\title{
Identification of gross deletions in FBN1 gene by MLPA
}

Hang Yang ${ }^{1 \dagger}$, Yanyun Ma ${ }^{1 \dagger}$, Mingyao Luo ${ }^{2}$, Kun Zhao $^{1}$, Yinhui Zhang ${ }^{1}$, Guoyan Zhu', Xiaogang Sun ${ }^{2}$, Fanyan Luo ${ }^{3}$, Lin Wang ${ }^{3^{*}}$, Chang Shu ${ }^{2^{*}}$ and Zhou Zhou ${ }^{1 *}$ (1)

\begin{abstract}
Background: Marfan syndrome (MFS) is an autosomal dominant connective tissue disorder caused by mutations in the FBN1 gene. Approximately 90\% of classic MFS patients have a FBN1 mutation that can be identified by singlegene sequencing or gene-panel sequencing targeting FBN1. However, a small proportion of MFS patients carry a large genomic deletion in FBN1, which cannot be detected by routine sequencing. Here, we performed an MLPA (multiplex ligation-dependent probe amplification) test to detect large deletions and/or duplications in FBN1 and TGFBR2 in 115 unrelated Chinese patients with suspected MFS or early-onset aneurysm/dissection.
\end{abstract}

Results: Five novel large deletions encompassing a single exon or multiple exons in the FBN1 gene were characterized in five unrelated patients, of which four were proven by Sanger sequencing, and the breakpoints were identified. Three of them met the revised Ghent criteria when genetic results were not available, and the other two patients were highly suspected and diagnosed with MFS until the FBN1 deletions were identified.

Conclusions: Our finding expands the mutation spectrum of large FBN1 deletions and emphasizes the importance of screening for large FBN1 deletions in clinical genetic testing, especially for those with classic Marfan phenotype.

Keywords: Marfan syndrome, MLPA, FBN1 gene, Deletion

\section{Background}

Marfan syndrome (MFS) is a connective tissue disorder with high clinical heterogeneity, mainly involving ocular, skeletal, and cardiovascular systems, with an estimated prevalence of 1:3000-1:5000 [1]. A large proportion of patients have visible signs, such as tall and slender stature, arachnodactyly, chest deformity, and scoliosis. Most patients have rapidly progressive myopia, and approximately $60 \%$ of affected individuals have ectopia lentis. However, cardiovascular abnormality might

\footnotetext{
* Correspondence: wanglin79922@csu.edu.cn;

changshu01@fuwaihospital.org; zhouzhou@fuwaihospital.org

${ }^{+}$Hang Yang and Yanyun Ma contributed equally to this work.

${ }^{3}$ Department of Cardiovascular Surgery, Xiangya Hospital Central South University, Changsha 410008, Hunan, China

${ }^{2}$ State Key Laboratory of Cardiovascular Disease, Center of Vascular Surgery, Fuwai Hospital, National Center for Cardiovascular Diseases, Chinese

Academy of Medical Sciences and Peking Union Medical College, Beijing 100037, China

'State Key Laboratory of Cardiovascular Disease, Beijing Key Laboratory for Molecular Diagnostics of Cardiovascular Diseases, Diagnostic Laboratory Service, Fuwai Hospital, National Center for Cardiovascular Diseases, Chinese Academy of Medical Sciences and Peking Union Medical College, Beijing 100037, China
}

be the only defect in some MFS patients that is insidious and fatal.

MFS is caused by mutations in the FBN1 gene, which is located on chromosome $15 \mathrm{q} 21.1$ and encodes a 320-kDa extracellular matrix glycoprotein fibrillin-1 [2, 3], a major component of microfibrils. So far, more than 2500 mutations (HGMD Professional 2018.1 total) have been identified throughout $F B N 1$, while missense mutations are the most common type [4,5]. Sanger sequencing of $F B N 1$ and panel sequencing including $F B N 1$ as well as a number of other genes associated with inherited aortopathies are commonly used to identify mutations [6]; however, both of these methods have a limitation for detecting FBN1 large deletions (del) or duplications (dup), which have been reported in up to 7\% of MFS patients [7].

Additionally, Loeys-Dietz syndrome (LDS), another inherited connective tissue disorder, which is caused mostly by TGFBR 1 and TGFBR 2 mutations, is often clinically indistinguishable from MFS [8]. However, up to now, no large genomic rearrangements in TGFBR1

(c) The Author(s). 2018 Open Access This article is distributed under the terms of the Creative Commons Attribution 4.0 International License (http://creativecommons.org/licenses/by/4.0/), which permits unrestricted use, distribution, and 
Table 1 The baseline clinical characteristics of the 115 unrelated patients

\begin{tabular}{ll}
\hline Characteristics & Statistics $(n=115)$ \\
\hline Age (years) & $29.4 \pm 14.9$ \\
Male gender & $87(75.7 \%)$ \\
Primary diagnosis & \\
Marfan syndrome & $19(16.5 \%)$ \\
Suspected Marfan syndrome & $43(37.4 \%)$ \\
Thoracic aortic aneurysm and dissection & $53(46.1 \%)$ \\
\hline Values are presented as mean \pm SD or $n(\%)$ &
\end{tabular}

Values are presented as mean \pm SD or $n(\%)$ or TGFBR2 have been reported in patients with aortic aneurysm/dissection and LDS features.

In this study, we performed a multiplex ligationdependent probe amplification (MLPA) testing of FBN1 and TGFBR2 in 115 unrelated Marfan or earlyonset aortopathy patients that were previously proven to be negative in a panel testing involving 15 genes associated with inherited aortopathy.

\section{Results}

A total of 115 patients with suspected MFS or earlyonset aortic aneurysm/dissection, who had a negative result in a 15-gene panel testing, were included in this study and evaluated for gross deletions and

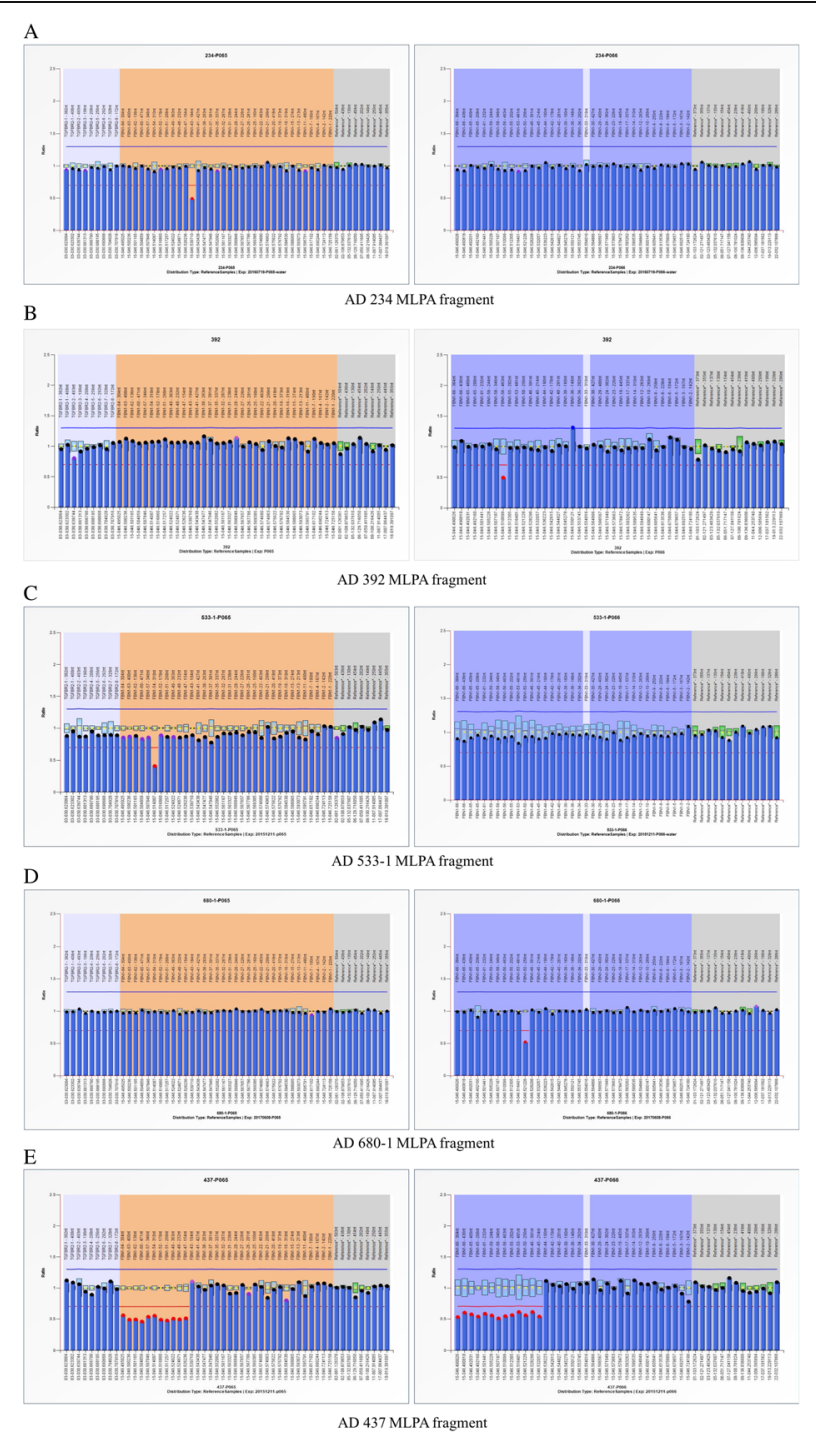

Fig. 1 Results of semiquantitative MLPA. The results of MLPA for five patients. a Reduced relative peak areas of FBN1 exon 43 for patient AD234. b Reduced relative peak areas of FBN1 exon 56 for patient AD392. c Reduced relative peak areas of FBN1 exon 54 for patient AD533-1. d Reduced relative peak areas of FBN1 exon 50 for patient AD680-1. e Reduced relative peak areas of FBN1 exon 44-66 for patient AD437 
Table 2 Overview of cases with large deletions in FBN1 gene

\begin{tabular}{lllll}
\hline Patient No. & Age (y) & Deletion breakpoints & $\begin{array}{l}\text { Deletion } \\
\text { (FBN1 exon affected) }\end{array}$ & Phenotype \\
\hline AD234 & 24 & g.48749026-48753819 & FBN1: exon 43 & Classic MFS \\
AD392 & 38 & g.48724560-48722281 & FBN1: exon 56 & Classic MFS \\
AD533-1 & 5 & g.48727672-48726338 & FBN1: exon 54 & Suspected MFS \\
AD680-1 & 14 & g.48734801-48730690 & FBN1: exon 50 & Suspected MFS \\
AD437 & 37 & NA & FBN1: exon 44-66 & Classic MFS \\
\hline All
\end{tabular}

All nucleotide positions are represented in relation to the human genome reference sequence (GRCh37/hg19), and position +1 corresponds to the first nucleotide of the FBN1 reference sequence (GenBank NC_000015.9) at the genomic DNA (g) level

NA not available duplications in FBN1 and TGFBR2 gene by MLPA assay. The baseline clinical characteristics are summarized in Table 1. Of all patients, 19 were classic MFS, which referred to those who met the Ghent criteria independent of genetic results, and 43 were suspected MFS, which referred to those with some positive signs (either aortic dilation or positive family history AND systemic score $\geq 3$ ) but not meeting the criteria yet. Almost half of the patients had no other systemic abnormality except for aortic events. Five novel large deletions encompassing a single exon or multiple exons in the $F B N 1$ gene were identified in five unrelated patients (Fig. 1, Table 2). Patients AD234, AD392, AD533-1, and AD680-1 harbored $F B N 1$ deletions of exon 43, exon 56, exon 54 , and exon 50, respectively, while patient AD437

A

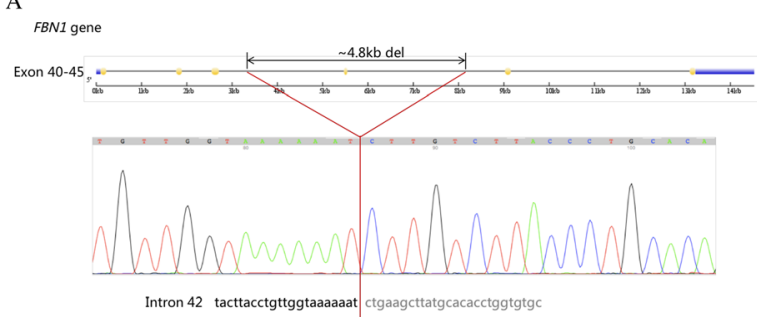

B

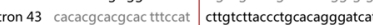

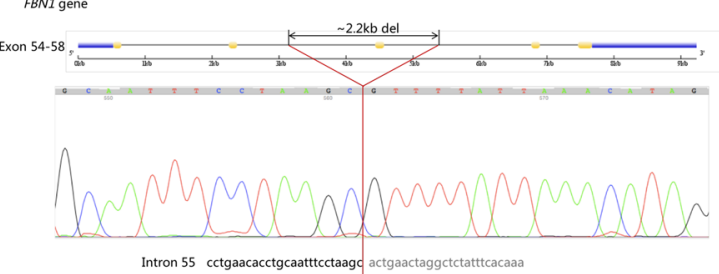

$\mathrm{C}$
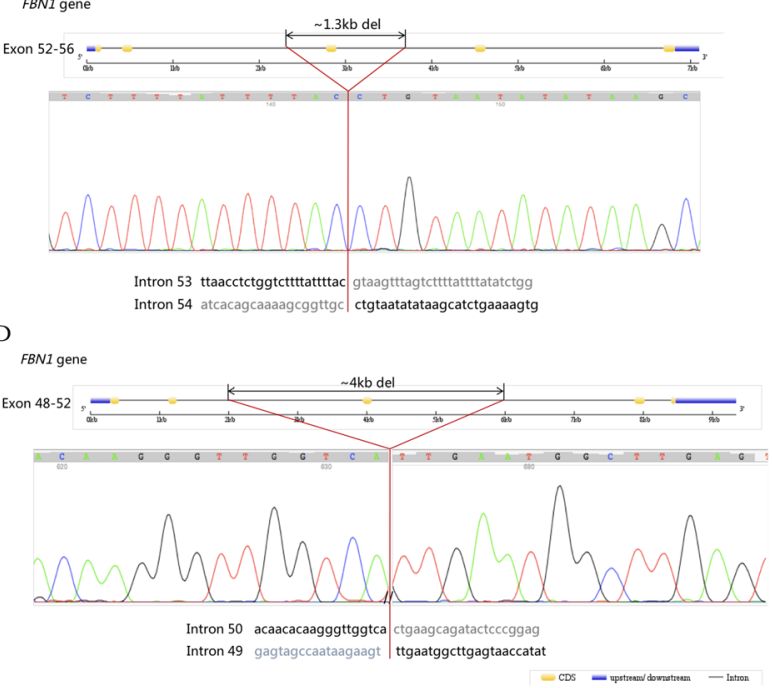

Fig. 2 Sequences of PCR products spanning the breakpoint junctions of the four single exon deletions. a $\sim 4.8 \mathrm{~kb}$ deletion encompassing exon 43 in patient AD234. $\mathbf{b} \sim 2.2$ kb deletion encompassing exon 56 in patient AD392. $\mathbf{c} \sim 1.3$ kb deletion encompassing exon 54 in patient AD533-1. d $~ 4.0 \mathrm{~kb}$ deletion encompassing exon 50 in patient AD680-1 
had a large deletion encompassing exons 44-66 in FBN1. These data had been submitted to ClinVar (ClinVar accessions SCV000804313-000804317).

To detect the breakpoints of deletions, we performed a long-range PCR followed by Sanger sequencing. Finally, the four single-exon deletions were all confirmed, and the breakpoints were found (Fig. 2). Regrettably, the deletion in AD437 could not be verified by the same method, since the mutated allele did not amplify well. Hence, we performed a quantitative PCR instead. Figure 3 shows that the quantity of genomic DNA from the proband amplified by primer pairs targeting exon 55 and exon 66 was half of that in the control samples, suggesting the true presence of a heterozygous deletion in this region.

All five FBN1 large deletion carriers had multiple system deformities. Information on the clinical manifestation of the disease and family history is summarized in Table 3. Three patients (AD234, AD392, and AD437) were classic MFS, while the other two (AD533-1 and AD680-1) both had a systemic score of 6 but did not meet the criteria yet when genetic results were not available, probably due to their young ages. Combined with genetic results, these two patients were eventually diagnosed with MFS. Notably, patient AD437 had a gross deletion involving the last 23 exons, but there was no significant difference in the severity of clinical phenotypes when compared with the other four single-exon deletion carriers.

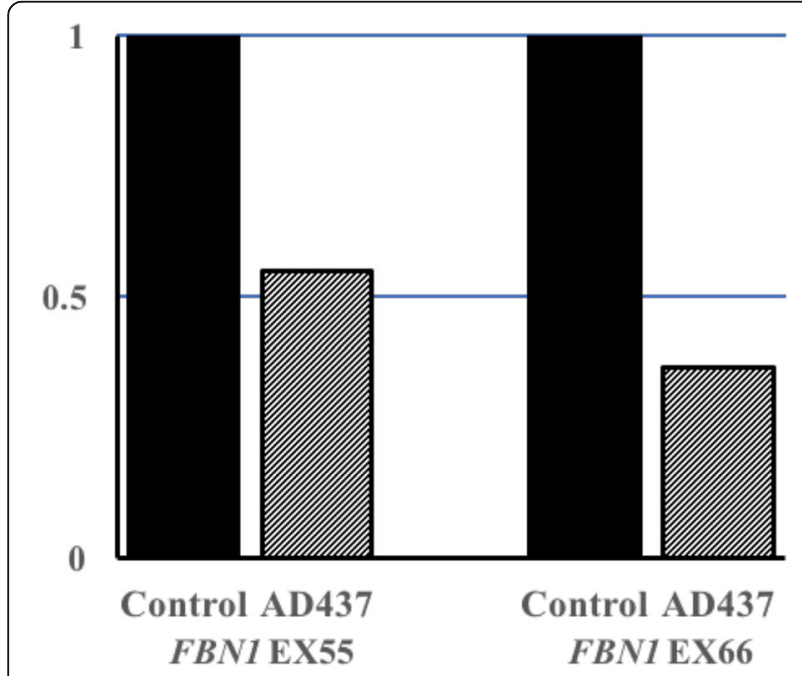

Fig. 3 Verification of gross deletions in AD437 by quantitative PCR. The bar graph shows the relative ratio of DNA from AD437, indicating the presence of a heterozygous deletion in the region
Table 3 The information of patients' clinical manifestation and family history

\begin{tabular}{|c|c|c|c|c|c|}
\hline & \multicolumn{5}{|c|}{ Patients } \\
\hline & AD234 & AD392 & $\begin{array}{l}\text { AD533- } \\
1\end{array}$ & $\begin{array}{l}\text { AD680- } \\
1\end{array}$ & AD437 \\
\hline Age (y) & 24 & 38 & 5 & 14 & 37 \\
\hline Gender & Male & Female & Male & Male & Female \\
\hline Height (cm) & 178 & 167 & 120 & 180 & 177 \\
\hline Weight (kg) & 70 & 58 & 23 & 52 & 71 \\
\hline \multicolumn{6}{|l|}{ Cardiovascular system } \\
\hline Aortic diameter $(\mathrm{cm})$ & 5.6 & 3.5 & 3.3 & 3.2 & 4.7 \\
\hline Z-score & 8.8 & 2.0 & 6.8 & 2.1 & 6.2 \\
\hline Aortic dissection & Y & N & N & $\mathrm{N}$ & Y \\
\hline \multicolumn{6}{|l|}{ Skeletal system } \\
\hline $\begin{array}{l}\text { Pectus carinatum } \\
\text { deformity }\end{array}$ & Y & NA & Y & $\mathrm{N}$ & Y \\
\hline Wrist and thumb signs & Y & Y & Y & Y & Y \\
\hline $\begin{array}{l}\text { Scoliosis or } \\
\text { thoracolumbar kyphosis }\end{array}$ & N & N & $N$ & Y & $\mathrm{N}$ \\
\hline Joint hypermobility & NA & Y & $\mathrm{N}$ & $\mathrm{N}$ & $\mathrm{N}$ \\
\hline $\begin{array}{l}\text { Reduced upper } \\
\text { segment/lower segment } \\
\text { ratio AND increased } \\
\text { arm/height }\end{array}$ & $\mathrm{N}$ & $\mathrm{N}$ & NA & Y & $\mathrm{N}$ \\
\hline Hindfoot deformity & NA & Y & $\mathrm{N}$ & $\mathrm{N}$ & NA \\
\hline \multicolumn{6}{|l|}{ Ocular } \\
\hline Ectopia lentis & $\mathrm{N}$ & Y & $\mathrm{N}$ & $\mathrm{N}$ & NA \\
\hline Myopia/strabismus & Y & Y & Y & Y & $\mathrm{N}$ \\
\hline \multicolumn{6}{|l|}{ Other features } \\
\hline Skin striae & $\mathrm{N}$ & Y & $N$ & $N$ & $Y$ \\
\hline Family history & Y & NA & $\mathrm{N}$ & $\mathrm{N}$ & Y \\
\hline
\end{tabular}

$Y$ presence of criterion, $N$ absence of criterion, $N A$ not available

\section{Discussion}

Marfan syndrome has a highly variable manifestation, from a mild phenotype to early-onset and rapidly progressive MFS. Cardiovascular abnormality could be the only defect in some affected individuals. According to the 2010 revised Ghent criteria, in the absence of family history, the combination of aortic root dilation $(Z \geq 2) /$ dissection and identification of a causal FBN1 mutation was sufficient to establish a diagnosis of MFS [9]. Accordingly, we performed an MLPA assay to screen for FBN1 and TGFBR2 large genomic rearrangements not only in the diagnosed/suspected MFS patients but also in those early-onset aneurysm/dissection patients with minor skeletal and ocular involvement, who had a negative result in a 15-gene panel testing associated with heritable aortopathy.

Finally, five patients with large $F B N 1$ deletions were identified in our cohort. All five patients had multiple systemic deformities, and three of them met the 2010 
Table 4 Overview of MFS cases with gross deletions in FBN1 gene

\begin{tabular}{|c|c|c|c|c|}
\hline \multicolumn{2}{|l|}{ Variation } & \multicolumn{2}{|l|}{ Patient } & \multirow{2}{*}{$\begin{array}{l}\text { Reference PMID } \\
\text { (year) }\end{array}$} \\
\hline Deletion (FBN1 exon affected) & Affected domains & Age $(y)$ & Phenotype in papers & \\
\hline \multicolumn{5}{|l|}{ Single-exon deletion } \\
\hline FBN1:g.46,701,985_46,728,871 (Ex1) & - & 25 & Classic MFS & $17492313(2002)$ \\
\hline$F B N 1: E \times 1$ & - & NA & Classic MFS & $24501682(2013)$ \\
\hline$F B N 1: E x 1$ & - & NA & Classic MFS & 24793577 (2014) \\
\hline$F B N 1: E \times 2$ & - & 52 & Classic MFS & $11700157(2001)$ \\
\hline FBN1:Ex3 & 1st EGF-like & NA & MFS & $21907952(2011)$ \\
\hline FBN1:Ex6 & 3rd EGF-like & 49 & Potential MFS & $28842177(2017)$ \\
\hline FBN1:c.3603_3668 del (Ex29) & 18th cbEGF-like & After birth & Neonatal MFS & 10441700 (1999) \\
\hline FBN1:Ex30 & 19-20th cbEGF-like & $<1$ & Suspected Beals-Hecht syndrome & $25944730(2015)$ \\
\hline FBN1:Ex32 & 21-22th cbEGF-like & 1 & Neonatal MFS & $18412115(2008)$ \\
\hline FBN1:Ex36 & 25-26th cbEGF-like & NA & Classic MFS & 19839986 (2009) \\
\hline FBN1:g.48,749,026_48,753,819 del (Ex43) & 7th TB, 29th cbEGF-like & 24 & Classic MFS & In this study \\
\hline FBN1:g.48,734,801-48,730,690 del (Ex50) & 35th cbEGF-like & 14 & MFS & In this study \\
\hline FBN1:Ex52 & 8th TB, 36th cbEGF-like & 40 & Classic MFS & $11700157(2001)$ \\
\hline FBN1:g.48,727,672-48,726,338 del (Ex54) & 37-38th cbEGF-like & 5 & MFS & In this study \\
\hline FBN1:g.48,724,560_48,722,281 del (Ex56) & 39-40th cbEGF-like & 38 & Classic MFS & In this study \\
\hline \multicolumn{5}{|l|}{ Multi-exon deletion } \\
\hline FBN1:Ex1-5 & 1-3rd EGF-like & 27 & Classic MFS & $21936929(2011)$ \\
\hline FBN1:g.46,580,456_46,883,035 (Ex1-16) & 1-3rd EGF-like, 1st TB, 4-10th cbEGF-like & 40 & Classic MFS & $17492313(2002)$ \\
\hline FBN1:Ex1-36 & 1-3rd EGF-like, 4-26th cbEGF-like, 1-5th TB & 15 & Classic MFS & $28842177(2017)$ \\
\hline FBN1:g.48,890,962_48,922,918 (Ex2-4) & 1-2nd EGF-like & 32 & Classic MFS & $29850152(2018)$ \\
\hline FBN1:Ex6-65 & 3rd EGF-like, 4-47th cbEGF-like, 1-9th TB & NA & Classic MFS & $24793577(2014)$ \\
\hline FBN1:Ex13-49 & 7-34th cbEGF-like, 3-7th TB & 5 & MFS & $18412115(2008)$ \\
\hline FBN1:Ex24-26 & 14-16th cbEGF-like & After birth & Neonatal MFS & $20455198(2010)$ \\
\hline FBN1:Ex33-38 & 21-26th cbEGF-like, 6th TB & 1 & Neonatal MFS & $24199744(2014)$ \\
\hline FBN1:Ex34-43 & 23-29th cbEGF-like, 6-7th TB & 22 & Classic MFS & $19863550(2010)$ \\
\hline FBN1:Ex37-65 & 26-47th cbEGF-like, 3-9th TB & NA & Classic MFS & $24793577(2014)$ \\
\hline FBN1:Ex42-43 & 7th TB, 29th cbEGF-like & $>46$ & Classic MFS & $11710961(2001)$ \\
\hline FBN1:Ex44-46 & 29-31th cbEGF-like & $>6$ & Childhood onset MFS & $11710961(2001)$ \\
\hline FBN1:Ex44-66 & 29-47th cbEGF-like, 8-9th TB & 37 & Classic MFS & In this study \\
\hline FBN1:Ex48-53 & 33-37th cbEGF-like, 8th TB & 15 & Neonatal MFS & $28842177(2017)$ \\
\hline FBN1:Ex49-50 & 34-35th cbEGF-like & 3 & Neonatal MFS & $28842177(2017)$ \\
\hline FBN1:Ex50-63 & 35-46th cbEGF-like, 8-9th TB & 65 & MFS & 19659760 (2009) \\
\hline FBN1:Ex58-63 & 41-46th cbEGF-like & 17 & Juvenile onset classic MFS & $17189636(2007)$ \\
\hline FBN1:c.7456_7821 del ${ }^{*}$ (Ex61-64) & 43-46th cbEGF-like & 48 & Classic MFS & 1631074 (1994) \\
\hline \multicolumn{5}{|l|}{ Whole gene deletion } \\
\hline FBN1:Ex1-66 & Full gene & 16 & Incomplete MFS & 20478419 (2010) \\
\hline FBN1:Ex1-66 & Full gene & 42 & Classic MFS & $21936929(2011)$ \\
\hline FBN1:Ex1-66 & Full gene & 15 & Classic MFS & 21936929 (2011) \\
\hline FBN1:Ex1-66 & Full gene & 12 & Classic MFS & 21936929 (2011) \\
\hline FBN1:Ex1-66 & Full gene & 41 & MFS & 21063442 (2011) \\
\hline FBN1:Ex1-66 & Full gene & 39 & MFS & 21063442 (2011) \\
\hline FBN1:Ex1-66 & Full gene & 16 & MFS & 21063442 (2011) \\
\hline FBN1:Ex1-66 & Full gene & 13 & MFS & 21063442 (2011) \\
\hline FBN1:Ex1-66 & Full gene & 27 & MFS & 21063442 (2011) \\
\hline FBN1:Ex1-66 & Full gene & 21 & MFS & 21063442 (2011) \\
\hline FBN1:Ex1-66 & Full gene & 34 & MFS & $21063442(2011)$ \\
\hline
\end{tabular}


Table 4 Overview of MFS cases with gross deletions in FBN1 gene (Continued)

\begin{tabular}{|c|c|c|c|c|}
\hline \multicolumn{2}{|l|}{ Variation } & \multicolumn{2}{|l|}{ Patient } & \multirow{2}{*}{$\begin{array}{l}\text { Reference PMID } \\
\text { (year) }\end{array}$} \\
\hline Deletion (FBN1 exon affected) & Affected domains & Age (y) & Phenotype in papers & \\
\hline$F B N 1: E x 1-66$ & Full gene & 5 & Potential MFS & $21063442(2011)$ \\
\hline FBN1:Ex1-66 & Full gene & 13 & Potential MFS & $21063442(2011)$ \\
\hline FBN1:Ex1-66 & Full gene & 8 & Potential MFS & $21063442(2011)$ \\
\hline FBN1:Ex1-66 & Full gene & 13 & Classic MFS & $22260333(2012)$ \\
\hline FBN1:g.48,931,968_51,102,375 (Ex1-66) & Full gene & 14 & MFS & 27615407 (2016) \\
\hline
\end{tabular}

NA not available

*The deletion was represented as nt. 4762 5127 in partial cloned sequence of FBN1 (PMID:1852207), and it was converted into its standardized nomenclature in accordance with HGVS (Human Genome Variation Society), in which the position +1 corresponds to the A of the ATG start codon of the mRNA reference sequence (GenBank NM_000138) at the cDNA (c) level. Except for this, all of the other nucleotide positions and patient phenotypes were shown as it was reported in the reference article

Ghent criteria when genetic results were not available, while the other two met the diagnostic criteria until $F B N 1$ gross deletions were detected, probably due to their young ages. Meanwhile, no gross deletions/duplications were identified in patients with only aortic aneurysm/dissection but without other systemic involvement. This result supported the hypothesis that FBN1 gross deletions usually lead to classic MFS [10-12].

Although gross genomic rearrangement within the FBN1 gene only contributed to a small proportion of MFS genetic causes (1.8-2.9\%) (UMD, http://www.umd.be/ FBN1/; HGMD, http://www.hgmd.cf.ac.uk/ac/gene.php?gene $=\mathrm{FBN} 1$ ), it was important to identify the pathogenic mutation to afford the patient an opportunity for prenatal testing and preimplantation genetic diagnosis (PGD). FBN1 mutations could be identified by sequencing in most Marfan patients (up to $93 \%$ in classic Marfan patients) [13]. However, Sanger sequencing and next-generation sequencing are commonly used in clinical genetic testing and are limited in their ability to detect large deletions and duplications. MLPA is a commonly used method to screen large del/dup, commercially, easily, and rapidly. In our cohort, 5 out of 62 patients $(8.1 \%)$ with diagnosed or suspected MFS but with negative results in panel sequencing had large $F B N 1$ deletions, which proved it to be efficacious and cost-effective to screen for FBN1 large genomic rearrangement in those MFS patients with multiple systemic involvements and a negative $F B N 1$ sequencing result.

Since MLPA and SNP (single-nucleotide polymorphism) arrays are more applicable in clinical genetic testing, increasing gross $F B N 1$ genomic deletions/duplications has been reported (summarized in Table 4), but until now, there has been no definite and conclusive genotype-phenotype correlation. Current studies reveal that the whole gene deletion of FBN1 did not lead to a more severe phenotype [12], and in-frame deletion involving exon 24-53 seemed to result in a high risk of early-onset and rapidly progressive form of MFS [11, 14-16].

\section{Conclusions}

In summary, our data expand the number of large FBN1 deletions and emphasize that screening for gross deletions in FBN1 genes is necessary for clinically suspected MFS patients, especially in those who have a negative result in conventional sequencing methods.

\section{Methods \\ Participants}

Patients with MFS or early-onset aortopathy were referred for a genetic test from the Center of Vascular Surgery in Fuwai Hospital and Department of Cardiovascular Surgery in Xiangya Hospital Central South University. Of these, 115 patients in whom no causal mutation was identified in a 15-gene panel associated with heritable aortopathy, including ACTA2, CLO3A1, FBN1, FBN2, MYH11, MYLK, NOTCH1, PRKG1, SKI, SLC2A10, SMAD3, SMAD4, TGFB2, TGFBR1, and $T G F B R 2$, were enrolled in this study to screen for $F B N 1$ and TGFBR2 large del/dup.

\section{Multiplex ligation-dependent probe amplification (MLPA)} MLPA assays were performed to detect $F B N 1$ and TGFBR2 large deletions or duplications using the commercially available SALSA MLPA kits P065 and P066 (MRC-Holland, Amsterdam, The Netherlands), which contained probes for all exons of FBN1 and TGFBR2. According to the manufacturer's instructions, a total of 100-200 ng of genomic DNA of each patient was used for hybridization, and amplification products from each MLPA assay were separated by capillary electrophoresis on an ABI 3500XL Dx Genetic Analyzer (Life Technologies, USA). The results were analyzed using Coffalyser software. Deletions and duplications with deviations more than $30 \%$ were suspected as significant alterations.

\section{Sanger sequencing}

To verify the results of MLPA and identify the breakpoints of the deletions, we performed a long-range PCR and subsequent Sanger sequencing. Primers flanking the 
predicted deletions were designed and LA Taq Hot Start Version kit (Takara, Japan) was used in the PCR system with the following cycling process: 5-min initial denaturation at $96{ }^{\circ} \mathrm{C}, 30$ cycles of $10 \mathrm{~s}$ at $98{ }^{\circ} \mathrm{C}$, and $15 \mathrm{~min}$ at $68{ }^{\circ} \mathrm{C}$, finished by a 10 -min final extension step at $72{ }^{\circ} \mathrm{C}$. Then, the products were detected through agarose gel electrophoresis and sequenced by the inner primers on the ABI 3730XL Genetic Analyzer.

\section{Quantitative PCR}

Quantitative PCR (qPCR) with the SYBR green reporter dye was performed to quantify relative target gene regions copy number in genomic DNA, and housekeeping gene GAPDH (glyceraldehyde 3-phosphate dehydrogenase) was used as the reference gene. The primer pairs were designed by Primer3 Input (http://bioinfo.ut.ee/primer3-0.4.0/) (see Additional file 1). All qPCRs were performed using $2 \times$ SYBR FAST qPCR Kit Master Mix (KAPA Biosystems, America) with the QuantStudio 6 Flex Real-Time PCR System.

\section{Additional file}

Additional file 1: Table S1. The quantitative PCR primer pairs for AD437. (PDF $132 \mathrm{~kb}$ )

\section{Abbreviations}

del: Deletion; dup: Duplication; FBN1: Fibrillin-1; GAPDH: Glyceraldehyde 3phosphate dehydrogenase; HGMD: Human Gene Mutation Database; HGVS: Human Genome Variation Society; LDS: Loeys-Dietz syndrome; MFS: Marfan syndrome; MLPA: Multiplex ligation-dependent probe amplification; NA: Not available; qPCR: Quantitative PCR; SNP: Single-nucleotide polymorphism; UMD: Universal Mutation Database

\section{Acknowledgements}

We thanked all the subjects who participated in this study.

\section{Funding}

This work was supported by the grant of CAMS Initiative for Innovative Medicine (2016-12M-1-016) and the grant from the Youth Foundation of Fuwai Hospital, National Center for Cardiovascular Disease, China (NO. 2016-F05).

\section{Availability of data and materials}

The datasets used and/or analyzed during the current study are available from the corresponding author on reasonable request.

\section{Authors' contributions}

HY coordinated the project and wrote the manuscript. YM and KZ performed MLPA and quantitative PCR. ML, XS, and FL recruited patients and collected clinical information. $Y Z$ and GZ were in charge of sample handling and quality control. LW and CS were in charge of the clinical evaluation and sample management. ZZ designed the project and revised the manuscript. All authors had read and approved the final manuscript.

\section{Ethics approval and consent to participate}

Everyone accepting the genetic test was adequately informed and signed a consent form. The study was approved by the ethics committee of Fuwai Hospital (Approval No. 2017-877) and adhered to the Declaration of Helsinki.

\section{Competing interests}

The authors declare that they have no competing interests.

\section{Publisher's Note}

Springer Nature remains neutral with regard to jurisdictional claims in published maps and institutional affiliations.

Received: 22 June 2018 Accepted: 18 September 2018

Published online: 04 October 2018

\section{References}

1. Judge DP, Dietz HC. Marfan's syndrome. Lancet (London). 2005;366(9501): 1965-76.

2. Keane $M G$, Pyeritz RE. Medical management of Marfan syndrome. Circulation. 2008;117(21):2802-13.

3. Maddox BK, Sakai LY, Keene DR, Glanville RW. Connective tissue microfibrils. Isolation and characterization of three large pepsin-resistant domains of fibrillin. J Biol Chem. 1989;264(35):21381-5.

4. Groth KA, Von Kodolitsch Y, Kutsche K, Gaustadnes M, Thorsen K, Andersen $\mathrm{NH}$, Gravholt $\mathrm{CH}$. Evaluating the quality of Marfan genotype-phenotype correlations in existing FBN1 databases. Genet Med. 2017;19(7):772-7.

5. Dordoni C, Ciaccio C, Santoro G, Venturini M, Cavallari U, Ritelli M, Colombi M. Marfan syndrome: report of a complex phenotype due to a 15q21.1 contiguos gene deletion encompassing FBN1, and literature review. Am J Med Genet A. 2017;173(1):200-6.

6. Yang H, Luo M, Fu Y, Cao Y, Yin K, Li W, Meng C, Ma Y, Zhang J, Fan Y, et al Genetic testing of 248 Chinese aortopathy patients using a panel assay. Sci Rep. 2016;6:33002.

7. Lerner-Ellis JP, Aldubayan SH, Hernandez AL, Kelly MA, Stuenkel AJ, Walsh J, Joshi VA. The spectrum of FBN1, TGFbetaR1, TGFbetaR2 and ACTA2 variants in 594 individuals with suspected Marfan syndrome, Loeys-Dietz syndrome or thoracic aortic aneurysms and dissections (TAAD). Mol Genet Metab. 2014;112(2):171-6.

8. Loeys BL, Chen J, Neptune ER, Judge DP, Podowski M, Holm T, Meyers J, Leitch CC, Katsanis N, Sharifi N, et al. A syndrome of altered cardiovascular, craniofacial, neurocognitive and skeletal development caused by mutations in TGFBR1 or TGFBR2. Nat Genet. 2005;37(3):275-81.

9. Loeys BL, Dietz HC, Braverman AC, Callewaert BL, De Backer J, Devereux RB, Hilhorst-Hofstee $Y$, Jondeau G, Faivre L, Milewicz DM, et al. The revised Ghent nosology for the Marfan syndrome. J Med Genet. 2010;47(7):476-85.

10. Furtado LV, Wooderchak-Donahue W, Rope AF, Yetman AT, Lewis T, Plant P, Bayrak-Toydemir P. Characterization of large genomic deletions in the FBN1 gene using multiplex ligation-dependent probe amplification. BMC Med Genet. 2011;12:119.

11. Li J, Wu W, Lu C, Liu Y, Wang R, Si N, Liu F, Zhou J, Zhang S, Zhang X. Gross deletions in FBN1 results in variable phenotypes of Marfan syndrome. Clin Chimica Acta. 2017:474:54-9.

12. Hilhorst-Hofstee $Y$, Hamel BC, Verheij JB, Rijlaarsdam ME, Mancini GM, Cobben JM, Giroth C, Ruivenkamp CA, Hansson KB, Timmermans J, et al. The clinical spectrum of complete FBN1 allele deletions. Eur J Human Genet. 2011;19(3):247-52.

13. Radonic T, de Witte $P$, Groenink $M$, de Bruin-Bon RA, Timmermans J, Scholte AJ, van den Berg MP, Baars MJ, van Tintelen JP, Kempers M, et al. Critical appraisal of the revised Ghent criteria for diagnosis of Marfan syndrome. Clin Genet. 2011:80(4):346-53.

14. Singh KK, Elligsen D, Liersch $R$, Schubert S, Pabst B, Arslan-Kirchner M, Schmidtke J. Multi-exon out of frame deletion of the FBN1 gene leading to a severe juvenile onset cardiovascular phenotype in Marfan syndrome. J Mol Cell Cardiol. 2007:42(2):352-6.

15. Liu W, Schrijver I, Brenn T, Furthmayr H, Francke U. Multi-exon deletions of the FBN1 gene in Marfan syndrome. BMC Med Genet. 2001;2:11.

16. Apitz C, Mackensen-Haen S, Girisch M, Kerst G, Wiegand G, Stuhrmann M, Niethammer K, Behrwind G, Hofbeck M. Neonatal Marfan syndrome: unusually large deletion of exons 24-26 of FBN1 associated with poor prognosis. Klinische Padiatrie. 2010;222(4):261-3.

\section{Consent for publication}

Everyone accepting the genetic test signed a consent form and agreed to allow their anonymized samples and genetic results to be used for further research studies and publications. 